CLINICAL STUDY

\title{
Preliminary data on biochemical remission of acromegaly after somatostatin analogs withdrawal
}

\author{
Cristina L Ronchi ${ }^{1,2}$, Erica Rizzo ${ }^{1,2}$, Andrea G Lania ${ }^{1,2}$, Rosario Pivonello ${ }^{4}$, Silvia Grottoli ${ }^{5}$, Annamaria Colao $^{4}$, \\ Ezio Ghigo ${ }^{5}$, Anna Spada ${ }^{1,2}$, Maura Arosio ${ }^{2,3}$ and Paolo Beck-Peccoz ${ }^{1,2}$ \\ ${ }^{1}$ Unit of Endocrinology, Fondazione IRCCS Ospedale Maggiore Policlinico, Mangiagalli e Regina Elena, Via F. Sforza, 35, 20122 Milan, Italy, ${ }^{2}$ Department \\ of Medical Sciences, University of Milan, 20122 Milan, Italy, ${ }^{3}$ Unit of Endocrinology, Osp. S. Giuseppe-Milanocuore AFaR, 20123 Milan, Italy, \\ ${ }^{4}$ Department of Molecular and Clinical Endocrinology and Oncology, Section of Endocrinology, Federico II University of Naples, 80131 Naples, Italy and \\ ${ }^{5}$ Division of Endocrinology and Metabolism, Department of Internal Medicine, San Giovanni Battista-Molinette Hospital, University of Turin, 10126 \\ Turin, Italy \\ (Correspondence should be addressed to C L Ronchi; Email: cristina.ronchi@unimi.it)
}

\begin{abstract}
Objective: It is still unknown whether prolonged treatment with somatostatin analogs (SSTa) may cause a long-lasting disease remission in GH-secreting adenomas after drug discontinuation. The aim of the present study was to investigate the evolution of GH/IGF-I secretion and tumor mass after SSTa withdrawal in patients affected by acromegaly.

Patients and Design: A total of 27 patients with acromegaly (12 de novo and 15 previously operated) were treated with SSTa for a median period of 48 months and considered optimally controlled in hormonal and neuroradiological terms. None of them were previously irradiated.

Methods: Basal GH, post-glucose GH nadir, IGF-I, clinical signs/symptoms, and metabolic parameters were evaluated after 12-16 weeks from drug withdrawal. Only patients who met the current criteria for disease remission remained in drug suspension being periodically re-evaluated for biochemical/clinical data and neuroradiological imaging.

Results: After 12-16 weeks withdrawal, 15 of the 27 patients had disease relapse and restarted SSTa, while 12 were considered 'in disease remission' ( $44 \%$ of total). Glucose metabolism improved in both euglycemic and diabetic patients after short-term SSTa discontinuation. Only one of the ten patients who reached 24 weeks withdrawal showed biochemical disease recurrence. On the whole, five of the patients still in remission after 6 months have already prolonged the follow-up over 12 months (median: 24 months), without clinical and biochemical/neuroradiological evidence of disease recurrence.

Conclusions: These preliminary data indicate a successful withdrawal of SSTa at least in a subset of wellresponsive patients with acromegaly and challenge the previously held concept that medical therapy is always a lifelong requirement.
\end{abstract}

European Journal of Endocrinology 158 19-25

\section{Introduction}

Acromegaly is an insidious disease caused by chronic growth hormone $(\mathrm{GH})$ and insulin-like growth factor-I (IGF-I) hypersecretion prevalently caused by a GH-secreting pituitary adenoma and associated with increased morbidity and mortality (1-3). Premature death is mainly due to a specific cardiomyopathy (4) worsened by the coexistence of several cardiovascular risk factors, such as abnormal carbohydrate metabolism and/or insulin resistance $(5,6)$. The objectives of treatment include normalization of GH/IGF-I secretion and control of pituitary tumor growth and acromegalyrelated comorbidities in order to normalize quality of life and mortality (7). To date, therapeutic options for acromegaly are surgical removal of pituitary adenoma and/or medical treatment with long-acting somatostatin analogs (SSTa), such as octreotide long-acting release (o-LAR) and lanreotide in both the slow-release formulation (l-SR) and the new Autogel formulation (l-ATG). It is well known that neurosurgery, when successful, normalizes life expectancy in acromegalic patients. Many studies also demonstrated that both primary and secondary prolonged treatments with SSTa are able to induce an enduring GH/IGF-I reduction and a pituitary tumor volume decrease in the majority of patients with acromegaly (8-10). However, to date, it is unknown whether SSTa may also provoke a longlasting disease remission in GH-secreting adenomas after drug discontinuation, similarly to the definitive cure of prolactinomas frequently induced by dopamine agonists $(11,12)$. 
The aim of the present study was to examine the evolution of GH/IGF-I secretion and tumor mass after drug withdrawal in a selected group of patients with acromegaly characterized by an optimal disease control during chronic treatment with different longacting SSTa.

\section{Materials and methods \\ Inclusion and exclusion criteria}

The inclusion and exclusion criteria had been discussed a priori on a multicenter basis by all three involved Italian Centers (Milan, Naples, and Turin).

Patients with a previous diagnosis of acromegaly were selected along the following inclusion criteria: first, the patients had to be primarily (de novo) or secondarily (previously operated on unsuccessfully) treated with different long-acting SSTa for a period of at least 12 consecutive months. All the patients who previously underwent neurosurgery were evaluated for fasting and post-glucose serum GH and IGF-I concentrations two months after their operation. Cases of isolated high IGF-I levels were re-evaluated after 3-4 months to confirm the disease activity before starting eventual SSTa therapy (13). Secondly, the patients had to be considered controlled by SSTa therapy, as indicated by GH levels (mean of at least three samples during saline infusion) $<2.5 \mu \mathrm{g} / \mathrm{l}$ and normal IGF-I levels for age (14) at a stable dosage of SSTa for at least nine consecutive months. Thirdly, pituitary tumor or residual tumor volume had to be invisible, reduced, disappeared or at least remained stable at magnetic resonance imaging during SSTa.

The exclusion criterion was previous radiotherapy or radiosurgery.

\section{Patients}

Following the inclusion and exclusion criteria, 27 patients with acromegaly (10 males and 17 females, mean age \pm s.D. $57 \pm 13$ years, body mass index (BMI) $28.0 \pm 3.6 \mathrm{~kg} / \mathrm{m}^{2}$ ) treated with SSTa for a median period of 48 months (mean: $61 \pm 44$ months, range: 12-156 months) were recruited for this study. In particular, 11 patients were treated with o-LAR at the dose of $10(n=4), 20(n=5)$, or $30 \mathrm{mg}(n=2)$ every 28 days; 9 with l-SR at the dose of $60 \mathrm{mg}$ every 28 days; and 7 with l-ATG at the dose of $120 \mathrm{mg}$ every $28(n=1)$, $42(n=3)$, or 56 days $(n=3)$. Twelve patients were primarily treated, while 15 previously underwent unsuccessful neurosurgery. Moreover, 8 patients were euglycemic, 12 had impaired glucose tolerance, and 7 had diabetes mellitus ( 4 treated with dietetic regimen, 1 with oral drugs, and 1 with combined insulin and oral drug therapy).
Clinical, hormonal, and neuroradiological data of all patients before and during chronic SSTa therapy are summarized in Table 1.

The Local Ethical Committees of the three involved Italian centers approved the protocol study, and patients gave their informed written consent to participate.

\section{Study protocol and assays}

The entire study protocol had been previously revised on a multicenter basis.

At the last follow-up visit during chronic SSTa therapy, just before the start of the study, all patients had basal serum GH levels (mean of at least three samples during saline infusion) evaluated by IFMA (AutoDelfia, Wallac OY, Turku, Finland) and serum IGF-I levels by RIA (Mediagnost, Tübingen, Germany), as described previously (15). Acromegaly-related symptoms and signs, such as headache, sweating, paraesthesiae, tiredness, and arthralgia, were investigated and graded 0 (absent), 1 (mild), 2 (moderate), and 3 (severe) by the same observer at each visit and the fourth finger size of left hand by jewellery rings. Neuroradiological investigation was also performed in the same period by the magnetic resonance imaging (MRI) of the pituitary region before and after gadolinium contrast.

All patients underwent a short-term drug withdrawal (12 weeks for o-LAR and l-SR and 16 weeks for l-ATG) and were then re-evaluated for clinical and hormonal parameters, including post-glucose (75 g, oral glucose tolerance test (OGTT)) GH nadir. Patients who simultaneously showed basal GH levels $<2.5 \mu \mathrm{g} / \mathrm{l}$, GH nadir $<1 \mu \mathrm{g} / \mathrm{l}$, and normal IGF-I concentrations for age (14) prolonged drug suspension with clinical and hormonal re-evaluation every 3 months and MRI every 6 months.

All the patients were also studied for a series of cardiovascular and metabolic parameters during SSTa and every 3 months after drug suspension. Clinical examinations included weight and height with calculation of BMI, systolic and diastolic blood pressure measured according to the World Health Organization International Society of Hypertension Guidelines. Periodical laboratory assessment included fasting and 2-h post-OGTT glucose and insulin levels, glycosylated hemoglobin (HbA1c), total cholesterol, high- and lowdensity cholesterol, and triglycerides. All these parameters were measured by standard procedures. Basal insulin sensitivity was investigated by the homeostasis model assessment (HOMA-S $\%=22.5 /$ fasting insulin $(\mathrm{mU} / \mathrm{l}) \times$ fasting glucose $(\mathrm{mmol} / \mathrm{l}))(16)$. Patients were considered with hypertension and/or dyslipidemia according to ATPIII criteria (17), while glucose tolerance was defined according to the new American Diabetes Association (ADA) guidelines (18). 
Table 1 Clinical, hormonal, and neuroradiological data of acromegalic patients before and during therapy with different long-acting somatostatin analogs (SSTa).

\begin{tabular}{|c|c|c|c|c|c|c|c|c|c|c|c|}
\hline \multirow[b]{2}{*}{$\begin{array}{l}\text { Patient } \\
\text { no. }\end{array}$} & \multirow[b]{2}{*}{ Sex } & \multirow[b]{2}{*}{ TNS } & \multicolumn{3}{|c|}{ Before SSTa } & \multirow[b]{2}{*}{$\begin{array}{c}\text { Type of } \\
\text { SSTa }\end{array}$} & \multirow[b]{2}{*}{$\begin{array}{c}\text { Months } \\
\text { of } \\
\text { therapy }\end{array}$} & \multirow[b]{2}{*}{ Age } & \multicolumn{3}{|c|}{ During SSTa } \\
\hline & & & $\begin{array}{c}\text { GH } \\
(\mu \mathrm{g} / \mathrm{l})\end{array}$ & $\begin{array}{c}\text { IGF-I } \\
(\mathrm{nmol} / \mathrm{l})\end{array}$ & Tumor mass & & & & $\begin{array}{c}\text { GH } \\
(\mu \mathrm{g} / \mathrm{l})\end{array}$ & $\begin{array}{c}\text { IGF-I } \\
(\mathrm{nmol} / \mathrm{l})\end{array}$ & Tumor mass \\
\hline 1 & $\mathbf{F}$ & Yes & 12.0 & 45 & Absent residual & O-LAR & 19 & 66 & 0.7 & 18 & Absent residual \\
\hline 2 & $M$ & Yes & 25.0 & 191 & Residual & o-LAR & 72 & 28 & 0.4 & 30 & Stable \\
\hline 3 & M & Yes & 5.5 & 196 & Residual & I-SR & 72 & 55 & 0.2 & 17 & Stable \\
\hline 4 & M & No & 5.0 & 55 & Macroadenoma & I-ATG & 48 & 64 & 1.9 & 33 & Stable \\
\hline 5 & M & Yes & 2.3 & 52 & Absent residual & O-LAR & 36 & 58 & 1.0 & 21 & Absent residual \\
\hline 6 & $\mathrm{M}$ & Yes & 2.0 & 63 & Absent residual & I-SR & 42 & 68 & 1.0 & 39 & Absent residual \\
\hline 7 & $\mathrm{~F}$ & Yes & 2.6 & 82 & Absent residual & I-SR & 60 & 47 & 1.7 & 23 & Absent residual \\
\hline 8 & $\mathrm{~F}$ & No & 7.7 & 95 & Microadenoma & I-SR & 90 & 73 & 1.2 & 14 & Disappeared \\
\hline 9 & $\mathbf{F}$ & Yes & 10.0 & 79 & Residual & O-LAR & 156 & 63 & 0.3 & 9 & Disappeared \\
\hline 10 & $\mathbf{F}$ & Yes & 2.7 & 69 & Absent residual & I-SR & 156 & 68 & 1.5 & 20 & Absent residual \\
\hline 11 & $\mathrm{M}$ & No & 5.5 & 67 & Microadenoma & o-LAR & 24 & 59 & 0.6 & 36 & Disappeared \\
\hline 12 & $\mathrm{~F}$ & Yes & 8.0 & 40 & Absent residual & I-SR & 84 & 45 & 1.5 & 15 & Absent residual \\
\hline 13 & M & Yes & 6.0 & 81 & Residual & o-LAR & 36 & 44 & 0.7 & 33 & Reduced \\
\hline 14 & $\mathrm{~F}$ & No & 6.6 & 54 & Microadenoma & I-ATG & 41 & 48 & 2.1 & 28 & Reduced \\
\hline 15 & $\mathrm{~F}$ & No & 4.0 & 71 & Microadenoma & I-ATG & 48 & 75 & 0.3 & 27 & Reduced \\
\hline 16 & $\mathrm{~F}$ & No & 5.8 & 67 & Microadenoma & I-SR & 120 & 71 & 1.5 & 22 & ND \\
\hline 17 & $\mathbf{F}$ & No & 8.4 & 70 & Microadenoma & I-SR & 12 & 36 & 1.7 & 20 & Stable \\
\hline 18 & $\mathrm{~F}$ & Yes & 3.1 & 63 & Residual & o-LAR & 24 & 64 & 0.5 & 18 & Stable \\
\hline 19 & $\mathrm{M}$ & Yes & 3.5 & 44 & Absent residual & o-LAR & 18 & 48 & 2.0 & 25 & Absent residual \\
\hline 20 & $\mathrm{~F}$ & No & 4.0 & 35 & Microadenoma & I-ATG & 18 & 59 & 0.3 & 26 & Stable \\
\hline 21 & $M$ & Yes & 1.8 & 85 & Residual & o-LAR & 18 & 35 & 1.4 & 26 & Stable \\
\hline 22 & $\mathrm{M}$ & No & 9.7 & 107 & Microadenoma & o-LAR & 72 & 56 & 0.8 & 38 & Disappeared \\
\hline 23 & $\mathrm{~F}$ & No & 1.3 & 47 & Microadenoma & o-LAR & 92 & 72 & 0.2 & 18 & Disappeared \\
\hline 24 & $\mathrm{~F}$ & No & 10.2 & 130 & Microadenoma & I-ATG & 12 & 64 & 1.2 & 27 & Disappeared \\
\hline 25 & $\mathrm{~F}$ & No & 17.0 & 55 & Microadenoma & I-ATG & 48 & 42 & 0.1 & 17 & Reduced \\
\hline 26 & $\mathrm{~F}$ & Yes & 8.6 & 94 & Residual & I-ATG & 150 & 62 & 2.4 & 22 & Reduced \\
\hline 27 & $\mathrm{~F}$ & Yes & 9.5 & 69 & Absent residual & I-SR & 60 & 62 & 0.8 & 11 & Stable \\
\hline
\end{tabular}

F, female; M, male; TNS, trans-naso-sphenoidal neurosurgery; o-LAR, octreotide long-acting release; I-SR, lanreotide slow release; I-ATG, lanreotide Autogel; ND, not done due to patient refusal. Bold represents five acromegalic patients in disease remission after long-term SSTa suspension (median: 24 months, range: $12-48$ months).

\section{Statistical analysis}

Data are expressed as mean \pm s.D., unless otherwise stated. IGF-I values were compared with an appropriate age-adjusted range, as reported previously (19), and expressed also as SDS. Nadir GH was defined as the lowest value at any time after OGTT. Data were tested for normality of distribution by Kolmogorov-Smirnov test and log transformed to obtain normal distribution when necessary. Statistical analysis was carried out using paired or unpaired Student's t-test, as appropriate. Linear regression analysis was performed to calculate correlation coefficients between different parameters. Values of $P<0.05$ were considered as statistically significant.

\section{Results}

\section{First follow-up (12-16 weeks withdrawal)}

At the first evaluation after 12-16 weeks of drug suspension, both serum GH and IGF-I levels significantly increased from $1.0 \pm 0.7$ to $2.0 \pm 1.3 \mu \mathrm{g} / \mathrm{l}(P<0.001)$ and from $24 \pm 8$ to $45 \pm 31 \mathrm{nmol} / \mathrm{l}$ (SDS: from $-0.04 \pm 1.1$ to $+2.8 \pm 3.9, P<0.001)$ respectively (Fig. 1). Serum IGF-I concentrations recorded during SSTa positively correlated with those found at the first visit after SSTa discontinuation $(P<0.05, r=0.44)$. Mean fourth finger size of left hand did not significantly change after drug suspension (data not shown). Similarly, all the evaluated metabolic parameters did not vary significantly, except for fasting glucose and HbA1c which significantly decreased in all patients, but in particular those with altered glucose metabolism during SSTa (from $6.7 \pm 1.3$ to $5.7 \pm 1.1 \mathrm{mmol} / \mathrm{l}$, $P<0.001$ and from $6.2 \pm 0.9$ to $6.1 \pm 0.6 \%, P<0.01$ respectively). Moreover, seven patients with impaired glucose tolerance during SSTa became normally tolerant after drug discontinuation.

When evaluating single patients, 15 showed a rapid disease relapse at the first visit. In particular, seven patients clearly had biochemically active disease, while the other eight showed discrepancies between different parameters (post-glucose $\mathrm{GH}$ nadir $<1 \mu \mathrm{g} / \mathrm{l}$ but high IGF-I levels or normal IGF-I levels but GH nadir $>1 \mu \mathrm{g} / \mathrm{l}$ ). Seven of these patients also reported a variable 

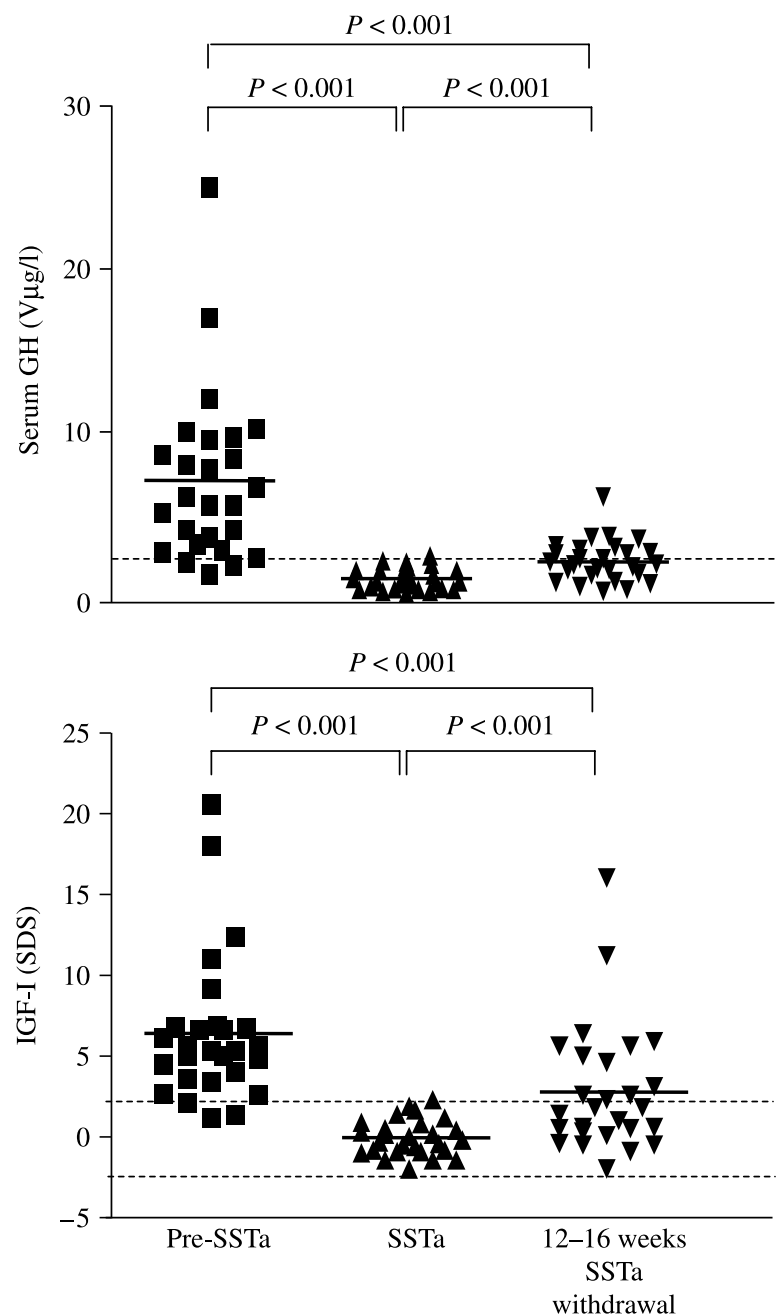

Figure 1 Mean serum GH and IGF-I concentration evaluated as standard deviation scores (SDS) before starting somatostatin analogues (pre-SSTa) during chronic long acting somatostatin analogues therapy (SSTa) and at the first visit after short term drug withdrawal (after 12-16 weeks) in all 27 acromegalic patients.

occurrence of acromegaly-related symptoms and signs (in particular arthralgia and soft tissue swelling). According to the study protocol, these 15 patients restarted SSTa therapy. The remaining 12 patients ( 1 male and 11 females, $44 \%$ of total) were considered to be in biochemical and clinical remission, showing the concomitant presence of all the three current criteria for cure (14) and absence of acromegaly-related symptoms and signs. Out of these 12 patients, 2 are still at 3 months suspension and are currently continuing withdrawal (patient no.s 20 and 23), while the remaining 10 have already been investigated after 6 months of SSTa suspension.

\section{Second follow-up (24 weeks withdrawal)}

Only one of the ten patients evaluated after 24 weeks withdrawal showed a biochemical disease recurrence with elevated basal GH levels despite still normal IGF-I concentrations and restarted SSTa therapy (patient no. 24). The remaining nine patients (33\% of total) were found to still be in biochemical and clinical remission (patient no.s 1, 5, 9, 10, 17, 18, 25, 26, and 27). These patients showed lower IGF-I levels recorded during SSTa treatment in comparison to the group of 16 patients with disease relapsing at first or second control visit $(-0.8 \pm 0.6$ vs $+0.4 \pm 1.1$, $P<0.05$; data not shown). As far as the neuroradiological imaging is concerned, one female previously operated patient showed a slight reduction of the residual tumor mass, while the other eight patients had a stable MRI panel. Subsequently, all of them further continued the follow-up.

\section{Long-term follow-up (over 12 months withdrawal)}

Out of the nine patients in remission after 6 months of SSTa withdrawal, five have already exceeded the followup from drug discontinuation by over 12 months ( 1 male and 4 females, $18.5 \%$ of total, patient no.s 1 , 5, 9, 10, and 17). In particular, one patient reached 18 months, three patients 24 months and one patient 48 months for a total median follow-up period of 24 months. All of them showed relatively stable hormonal levels and absence of clinical/neuroradiological disease recurrence and are currently continuing drug suspension. The trend of serum IGF-I data recorded in these patients during monitoring is described in Fig. 2.

All the historical, hormonal, and neuroradiological characteristics of these five patients in remission after long-term withdrawal are reported in Table 1. In particular, no differences in terms of age, previous treatments, type and duration of SSTa therapy, GH and IGF-I levels before SSTa treatment, fasting GH, and

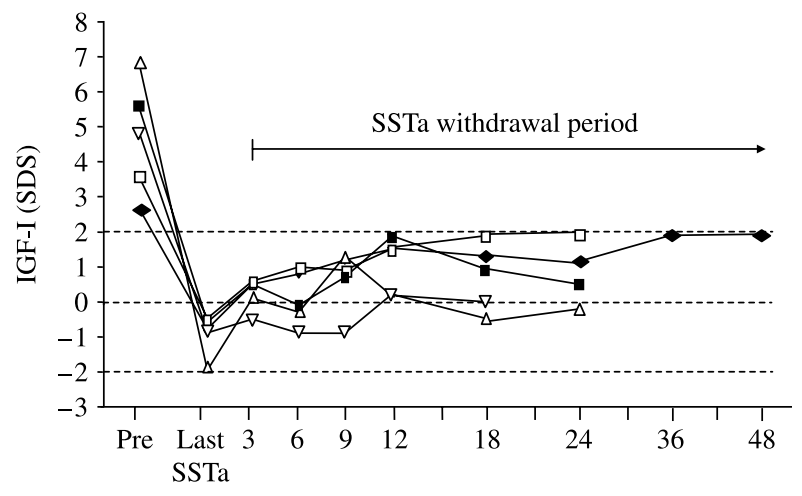

Time (months)

Figure 2 Serum IGF-I concentrations evaluated as standard deviation scores (SDS), recorded during somatostatin analogues therapy and at each visit after drug withdrawal in the 5 acromegalic patients considered in remission (normal IGF-I concentrations and nadir $\mathrm{GH}<\mathrm{I} \mu \mathrm{g} / \mathrm{I}(14)$ ) at the long-term follow-up (over at least 12 months of drug withdrawal). 
post-glucose $\mathrm{GH}$ nadir after drug suspension were observed in respect to other patients.

At the last visit, in these five patients (three affected by DM, one by IGT, and one euglycemic), fasting glucose levels continued to be observed as significantly lower compared to those observed during SSTa $(5.8 \pm 1.5$ vs $7.8 \pm 1.4 \mathrm{mmol} / \mathrm{l}, \quad P<0.005)$, while HbA1c levels remained only slightly lower $(6.3 \pm 0.9$ vs $6.9 \pm 1.1 \%$, $P=\mathrm{NS}$ ), despite steady BMI levels and the same antidiabetic therapy (two following dietetic regimen and one on insulin plus metformin). All the other evaluated metabolic parameters remained substantially stable during follow-up. Fourth finger size of left hand did not significantly change over the time. As far as MRI was concerned, one de novo patient showed the progressive occurrence of an intratumoral imaging compatible with an hemorrhagical area (patient no. 17), while none of the remaining patients developed any visible change of pituitary tumor or residual tumor mass during the long-term drug suspension.

\section{Discussion}

This preliminary study demonstrates that chronic treatment with long-acting SSTa may be able to induce a long-term disease remission at least in a subgroup of patients with acromegaly highly sensitive to SSTa therapy. In fact, a long-term persistent biochemical disease control after SSTa withdrawal (over 12 months) was achieved in about $20 \%$ of the 27 patients selected according to the presence of constantly 'safe' GH levels, normal IGF-I concentrations, and stable neuroradiological imaging during treatment. This hormonal remission was also accompanied by a persistent clinical well-being and an unchanged tumor/residual tumor volume, challenging the held notion that pharmacological therapy is always a permanent necessity. Moreover, a higher percentage of patients (about 40\%) showed at least a short-term biochemical and clinical disease control after drug discontinuation. This last finding also suggests the possibility of a scheduled periodical 3-6 month duration SSTa suspension without inducing any hormonal deterioration.

The mechanisms responsible for the long-term control of hormone secretion and tumor growth induced by SSTa therapy are at present unknown. However, SST has been shown to be a powerful inhibitor of neovascularization, by acting directly on endothelial cells or indirectly on the production of angiogenic growth factors (20). Furthermore, previous studies indicated that somatostatin and its analogs exert antiproliferative effects with cytostatic (growth arrest) and cytotoxic (apoptosis) consequences $(21,22)$. Consistent with these data, it is tempting to speculate that the long-term remission observed in a subset of patients might be due to the antiangiogenic and antiproliferative actions of SSTa, although further basic research is required to clearly understand this phenomenon.

Some previous studies conducted in small groups of patients demonstrated the possibility of prolonging intervals between o-LAR injection to every 6-8 weeks without reduction in efficacy $(23,24)$. In addition, at least two further papers evaluated the duration of o-LAR or l-SR suspension required to obtain a correct washout, fixing it at about 3 months $(25,26)$. By contrast, only one study specifically investigated a longterm SSTa suspension and reported safe GH and normal IGF-I levels after 5 months withdrawal in one of the seven patients well responsive to 12 months o-LAR therapy (27). The present study first demonstrates the possibility to obtain a long-lasting remission of acromegaly after a long-term SSTa suspension. Moreover, it suggests at least the need for a periodical SSTa discontinuation to evaluate the disease evolution in each patient, particularly in those well controlled by SSTa therapy. In view of programming SSTa discontinuation and follow-up intervals, it is worth noting that recurrence within 3 months from drug suspension was seen in almost all patients, while among those who prolonged the monitoring only one showed disease reappearance after 6 months withdrawal. In fact, in all the other monitored patients, no clear recurrence during the follow-up period (up to 6 months in four patients, 18 months in one, 2 years in three, and 4 years in one) was observed. Admittedly, the number of patients included in this series is still too small and the period of follow-up too short to draw definitive conclusions.

As far as the metabolic parameters were concerned, it is important to consider that glucose homeostasis showed some changes during the suspension period. In fact, glucose metabolism clearly and rapidly improved after short-term withdrawal in all patients, closely mirroring the absence of detrimental insulin inhibition by SSTa. Moreover, even during the long-term revaluation of patients in biochemical remission, fasting glucose levels remained steadily lower than those recorded during SSTa.

All subjects who resulted in remission after withdrawal appeared to have at least two specific common characteristics. First, a majority of five patients in longterm remission (more than 12 months) were previously operated by unsuccessful neurosurgery except one de novo patient (one showing the occurrence of hemorrhagical intratumoral area after 12 months of SSTa withdrawal). This observation is consistent with previous data demonstrating that about $75-80 \%$ removal of the tumor by neurosurgery (debulking) improves hormonal control of acromegaly by SSTa $(28,29)$. Secondly, the patients in median term remission (24 weeks) showed IGF-I concentrations lower than those found in other patients also during SSTa treatment. Thus, this study further confirms that IGF-I is the most important biochemical parameter to monitor during 
SSTa therapy, and provides new evidence that it may be considered the main predictive parameter of a longlasting disease control after drug withdrawal. On the contrary, no other differences in terms of age, previous neurosurgery, duration and type of SSTa therapy, GH and IGF-I levels before SSTa treatment, and GH levels after drug suspension were observed.

In conclusion, chronic SSTa therapy seems to be able to induce a long-term hormonal and clinical disease remission at least in a subgroup of carefully selected patients with acromegaly previously found to be highly sensitive to SSTa therapy. In this respect, IGF-I levels during SSTa treatment seem to be the main predictive parameter of disease control even after drug withdrawal. These data challenge the previously held concept that medical therapy is always a lifelong requirement, even if a close follow-up must be performed over the time and the resumption of treatment must be restarted whenever necessary.

\section{Acknowledgements}

The authors are grateful to all the nursing staff for their expert and kind help. The present study was partially supported by the research grants from Associazione Italiana Ricerca sul Cancro (AIRC) and from Fondazione IRCCS Ospedale Maggiore Policlinico, Mangiagalli e Regina Elena (Milan, Italy).

\section{References}

1 Melmed S. Acromegaly. New England Journal of Medicine 1990322 966-977.

2 Orme SM, McNally RJ, Cartwright RA \& Belchetz PE. Mortality and cancer incidence in acromegaly: a retrospective cohort study. United Kingdom Acromegaly Study Group. Journal of Clinical Endocrinology and Metabolism $1998 \mathbf{8 3} 2730-2734$.

3 Holdaway IM, Rajasoorya RC \& Gamble GD. Factors influencing mortality in acromegaly. Journal of Clinical Endocrinology and Metabolism 200489 667-674.

4 Clayton RN. Cardiovascular function in acromegaly. Endocrine Reviews $200324272-277$.

5 Foss MC, Saad MJA, Paccola GMGF, Paula FJA, Piccinato CE \& Moreira AC. Peripheral glucose metabolism in acromegaly. Journal of Clinical Endocrinology and Metabolism 199172 1048-1053.

6 Hansen I, Tsalikian E, Beaufrere B, Gerich J, Haymond M \& Rizza R. Insulin resistance in acromegaly: defects in both hepatic and extrahepatic insulin action. American Journal of Physiology 1986 250 E269-273.

7 Melmed S, Casanueva FF, Cavagnini F, Chanson P, Frohman L, Grossman A, Ho K, Kleinberg D, Lamberts S, Laws E, Lombardi G, Vance ML, Werder KV, Wass J \& Giustina A. Acromegaly Treatment Consensus Workshop Participants. Guidelines for acromegaly management. Journal of Clinical Endocrinology and Metabolism 2002 87 4054-4058.

8 Freda PU, Katznelson L, van der Lely AJ, Reyes CM, Zhao S \& Rabinowitz D. Long-acting somatostatin analog therapy of acromegaly: a meta-analysis. Journal of Clinical Endocrinology and Metabolism $2005904465-4473$.
9 Bevan JS. Clinical review: the antitumoral effects of somatostatin analog therapy in acromegaly. Journal of Clinical Endocrinology and Metabolism 200590 1856-1863.

10 Racine MS \& Barkan AL. Somatostatin analogs in medical treatment of acromegaly. Endocrine 200320 271-278.

11 Biswas M, Smith J, Jadon D, McEwan P, Rees DA, Evans LM, Scanlon MF \& Davies JS. Long-term remission following withdrawal of dopamine agonist therapy in subjects with microprolactinomas. Clinical Endocrinology 200563 26-31.

12 Colao A, Di Sarno A, Cappabianca P, Di Somma C, Pivonello R \& Lombardi G. Withdrawal of long-term cabergoline therapy for tumoral and nontumoral hyperprolactinemia. New England Journal of Medicine $20033492023-2033$.

13 Feelders RA, Bidlingmaier M, Strasburger CJ, Janssen JA, Uitterlinden P, Hofland LJ, Lamberts SW, van der Lely AJ \& de Herder WW. Postoperative evaluation of patients with acromegaly: clinical significance and timing of oral glucose tolerance testing and measurement of (free) insulin-like growth factor I, acid-labile subunit, and growth hormone-binding protein levels. Journal of Clinical Endocrinology and Metabolism $2005906480-6489$.

14 Giustina A, Barkan A, Casanueva FF, Cavagnini F, Frohman L, Ho K, Veldhuis J, Wass J, Von Werder K \& Melmed S. Criteria for cure of acromegaly: a consensus statement. Journal of Clinical Endocrinology and Metabolism 200085 526-529.

15 Ronchi CL, Varca V, Giavoli C, Epaminonda P, Beck-Peccoz P, Spada A \& Arosio M. Long-term evaluation of postoperative acromegalic patients in remission with previous and newly proposed criteria. Journal of Clinical Endocrinology and Metabolism $2005901377-1382$.

16 Matthews DR, Hosker JP, Rudenski AS, Naylor BA, Treacher DF \& Turner RC. Homeostasis model assessment: insulin resistance and $\beta$-cell function from fasting plasma glucose and insulin concentration in man. Diabetologia $1985 \mathbf{2 8} 412-419$.

17 Expert panel on detection, evaluation, and treatment of high blood cholesterol in adults. Executive summary of the third report of the national cholesterol education program (NCEP) expert panel on detection, evaluation, and treatment of high blood cholesterol in adults (ATPIII). Journal of the American Medical Association 2001 287 356-359.

18 American Diabetes Association. Diagnosis and classification of diabetes mellitus. Diabetes Care 200629 S43-48.

19 Epaminonda P, Porretti S, Cappiello V, Faglia G \& Arosio M. Efficacy of radiotherapy in normalizing serum IGF-I, acid-labil subunit (ALS) and IGF-BP3 levels in acromegaly. Clinical Endocrinology 200155 183-189.

20 Dasqupta P. Somatostatin analogues: multiple roles in cellular proliferation, neoplasia, and angiogenesis. Pharmacology and Therapeutics $2004 \mathbf{1 0 2} 61-85$.

21 Ferrante E, Pellegrini C, Bondioni S, Peverelli E, Locatelli M, Gelmini P, Luciani P, Peri A, Mantovani G, Bosari S, Beck-Peccoz P, Spada A \& Lania A. Octreotide promotes apoptosis in human somatotroph tumor cells by activating somatostatin receptor type 2. Endocrine-Related Cancer 200613 955-962.

22 Hubina E, Nanzer AM, Hanson MR, Ciccarelli E, Losa M, Gaia D, Papotti M, Terreni MR, Khalaf S, Jordan S, Czirják S, HanzélY Z, Nagy GM, Góth MI, Grossman AB \& Korbonits M. Somatostatin analogues stimulate p27 expression and inhibit the MAP kinase pathway in pituitary tumours. European Journal of Endocrinology $2006155371-379$.

23 van Thiel SW, Romijn JA, Biermasz NR, Ballieux BE, Frolich M, Smit JW, Corssmit EP, Roelfsema F \& Pereira AM. Octreotide longacting repeatable and lanreotide Autogel are equally effective in controlling growth hormone secretion in acromegalic patients. European Journal of Endocrinology 2004150 489-495.

24 Gilroy JJ \& James RA. Optimizing somatostatin analog therapy in acromegaly: long-acting formulations. Treatments in Endocrinology 2002 1 149-154 (Review).

25 Stewart PM, Stewart SE, Clark PM \& Sheppard MC. Clinical and biochemical response following withdrawal of a long-acting, depot injection form of octreotide (Sandostatin-LAR). Clinical Endocrinology 199950 295-299. 
26 Caron P, Tabarin A, Cogne M, Chanson P \& Jaquet P. Variable growth hormone profiles following withdrawal of long-term $30 \mathrm{mg}$ slowrelease lanreotide treatment in acromegalic patients: clinica implications. European Journal of Endocrinology 2000142 565-571.

27 Lorcy Y, Dejager S, Chanson P \& French Octreotide LAR Group. Time course of GH and IGF-1 levels following withdrawal of longacting octreotide in acromegaly. Pituitary 20003 193-197.

28 Petrossians P, Borges-Martins L, Espinoza C, Daly A, Betea D, Valdes-Socin H, Stevenaert A, Chanson P \& Beckers A. Gross total resection or debulking of pituitary adenomas improves hormonal control of acromegaly by somatostatin analogs. European Journal of Endocrinology 2005152 61-66.
29 Colao A, Attanasio R, Pivonello R, Cappabianca P, Cavallo LM, Lasio G, Lodrini A, Lombardi G \& Cozzi R. Partial surgical removal of growth hormone-secreting pituitary tumors enhances the response to somatostatin analogs in acromegaly. Journal of Clinical Endocrinology and Metabolism 200691 85-92.

Received 27 September 2007

Accepted 8 October 2007 